\title{
Temperament and Eating Attitudes in an Adolescent Community Sample: A Brief Report
}

\author{
Enrica Marzola, Secondo Fassino, Federico Amianto, and Giovanni Abbate-Daga \\ Eating Disorders Center, Department of Neuroscience, University of Turin, Via Cherasco 15, 10126 Turin, Italy \\ Correspondence should be addressed to Giovanni Abbate-Daga; giovanni.abbatedaga@unito.it
}

Received 3 June 2014; Revised 25 August 2014; Accepted 27 August 2014; Published 8 September 2014

Academic Editor: Xingguang Luo

Copyright (C) 2014 Enrica Marzola et al. This is an open access article distributed under the Creative Commons Attribution License, which permits unrestricted use, distribution, and reproduction in any medium, provided the original work is properly cited.

\begin{abstract}
Objective. Temperament traits like high harm avoidance (HA) have been proposed as putative risk factors for the development of eating disorders (EDs). We aimed at studying the relationship between temperament and eating attitudes on a large community sample of adolescents. Method. We recruited 992 high school students aged 14-18. In addition to measuring body mass index (BMI), participants were asked to complete the temperament and character inventory and the food frequency questionnaire. Results. Sixtytwo percent of the sample reported overeating, 22.8\% reported normal eating, and $15.2 \%$ reported under eating. Under and normal eaters had higher BMI than that of over eaters. Harm avoidance was found to be significantly higher in those participants with lower eating intakes whilst novelty seeking was found to be higher in over eaters. Conclusion. An interesting association between temperament (high HA) and food approach (under eating) emerged. Longitudinal studies are needed to evaluate whether these traits represent a risk factor for the development of EDs.
\end{abstract}

\section{Introduction}

Eating disorders (EDs) are serious illnesses characterized by high comorbidity and mortality, life-threatening sequelae, and low quality of life. Notwithstanding the recent insights into the mechanisms underpinning and maintaining such disorders, to date their aetiology remains unknown and uncertainty persists on what to consider as vulnerability factors.

Among various other posited factors, personality has been called into question as regards ED developmental trajectories. According to the widely used instrument temperament and character inventory (TCI) [1], high scores on the temperament dimension of harm avoidance (HA) are shared by both actively ill and recovered individuals [2]; although its role is not univocal, these lines of research seem to propose it as candidate risk factor for the development of EDs [3].

More in detail, the rationale for considering personality as risk factor could be represented by its role in modulating coping abilities, namely, those responses based on personal vulnerability to stressors that are shared by many individuals. Earlier studies showed how the combination of high HA and low self-directedness may impair the ability to face distress
[4] and recent research proposed that personality traits may indirectly influence eating attitudes and food approach through thin-ideal internalization [5].

In addition to an earlier study [6] focusing on disordered eating in adolescents, with the present report we aimed to assess the relationship between temperament traits and eating attitudes on a large community sample of adolescents. In line with the pattern of personality traits-as measured by the TCI-characterizing patients affected by EDs (i.e., particularly high HA) $[4,7]$ our a priori hypothesis was to find an association between a highly avoidant temperament and food approach.

\section{Methods}

2.1. Participants. Methods are described in detail elsewhere [6]. Ten high schools in Turin were randomly selected and asked to participate in this study; of these, six agreed on being involved. We finally enrolled 992 students $(N=247$ males; $N=745$ females), aged $14-18$ years old. No exclusion/ inclusion criteria were adopted. All students were Caucasian and assessed while being at school. 
TABLE 1: Comparison of personality characteristics as measured with the TCI among caloric intake groups according to the food frequency questionnaire.

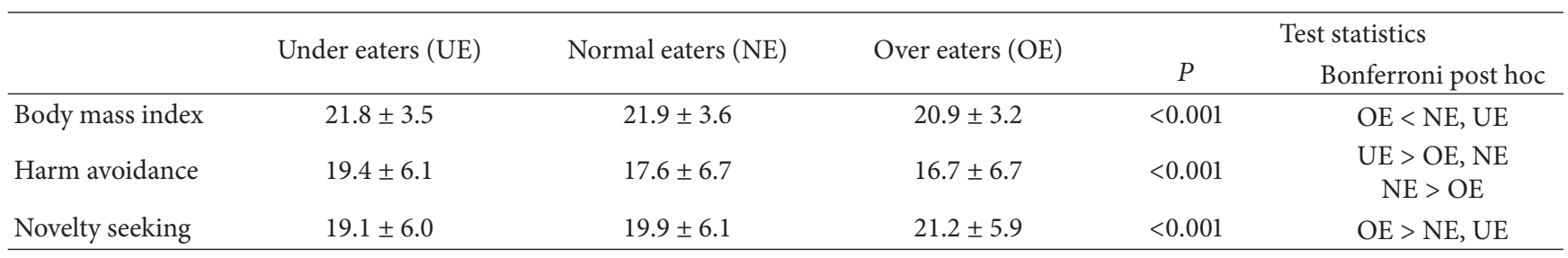

All participants over the age of 18 gave their written informed consent; those under the age of 18 provided written informed assent in addition to their parents' written informed consent and permission. All consent/assent procedures were conducted according to the ethical committee of the Department of Neuroscience of the University of Turin.

2.2. Measures. All participants' weight and height were measured by a registered dietician and body mass index (BMI) was then calculated. All students were asked to provide their personal information and to complete the following self-reported questionnaires: (1) temperament and character inventory (TCI) [1] to evaluate personality according to seven dimensions (temperament: novelty seeking [NS], harm avoidance [HA], reward dependence [RD], and persistence $[\mathrm{P}]$; and character: self-directedness [SD], cooperativeness $[\mathrm{C}]$, and self-transcendence [ST]); and (2) food frequency questionnaire (FFQ) [8] to measure individuals' usual food and caloric intake. Norms are based on the nutritional recommendations (adjusted for age and gender) for the Italian population described by the Italian Society of Human Nutrition [9]. According to such recommendations, subjects can be divided into 3 groups: under eaters (UE), normal eaters $(\mathrm{NE})$, and over eaters (OE).

2.3. Statistical Analysis. The SPSS statistical software package was used for data analysis. Descriptive statistics were computed. A one-way analysis of variance (ANOVA) with Bonferroni post hoc was calculated to assess personality dimensions by eating attitudes.

\section{Results}

Details on data collection have been described elsewhere [6]; 992 adolescents were contacted, 972 were enrolled, and 967 returned the questionnaires; of these, 860 completed all the assessments. Participants' mean age was $16.2 \pm 1.5$ years.

As regards food intake, the majority of the sample (62\%) reported overeating, $22.8 \%$ normal eating, and $15.2 \%$ under eating whilst with respect to BMI, UE, and NE groups were found to have a significantly higher BMI than OE.

With respect to personality, harm avoidance (HA) was significantly higher $(P<0.001)$ in those participants with lower eating intakes when compared to other groups whilst novelty seeking (NS) was found to be higher in over eaters $(P<0.001)$ than that in the other groups (see Table 1$)$.

\section{Discussion}

Interestingly, under eaters showed a temperament profile similar to individuals affected by EDs, scoring higher on the harm avoidance scale when compared to normal and over eaters. Harm avoidance is a temperament dimension related to behavioral inhibition, anxiety, fearfulness, pessimism, and inflexibility and it has been widely demonstrated to be high in patients affected by EDs $[2,4]$. As extensively reported in literature [7], HA levels are connected to the serotonin system in the brain, namely, the neurobiological substratum that is strictly related to mood, impulse control, and satiety.

Individuals affected by anorexia nervosa starve themselves to death and robust evidence has now accumulated suggesting that starvation per se could contribute to modulate dysphoric mood potentially generated by an increased activity of brain serotonin systems [7]. The association we found between high HA and under eating in healthy adolescents raises the intriguing hypothesis that also for certain individuals from the community lower caloric intakes may represent an attempt to attenuate aversive anxious and dysphoric states related to a highly avoidant temperament.

Moreover, a mismatch between self-reported eating attitudes and BMI emerged since under eaters reported low caloric intakes although their BMI was found to be higher than that of over eaters. On one hand this finding may be due to young participants' lack of accuracy in self-evaluation or age-related different metabolic requirements but on the other hand it could describe instead under eaters' peculiar food approach. In fact, it could be speculated that such tendency to underestimate energy intakes may be explained by factors like thin-ideal internalization and body dissatisfaction. Such factors have been suggested to promote the ED onset [10] and also recently they have been proposed as mediators between personality and food approach [5].

As regards novelty seeking, higher levels on this dimension corresponded to larger food intakes. Also this datum is in line with the ED literature with lower NS scores in individuals who restrict their eating rather than in those who engage in binge-purging behaviors [4].

This report suffers from several limitations: participants were not screened for psychiatric comorbidities potentially biasing HA levels and disordered eating could have been assessed with more powerful tools. Moreover, food intake can be influenced by multiple factors including stress, mood, and general health which should be controlled for. However, the match we found between temperament traits of clinical and community samples is of interest in the context of the 
ongoing debate on developmental risk factors for EDs. Further studies with a longitudinal design may want to ascertain as to whether some temperament traits may constitute a slippery slope for predisposed individuals towards full-blown EDs through the impairment of coping abilities. Still, larger studies are warranted to shed light on the mismatch we found between self-report food intake and BMI.

Prompted by the need of distress modulation, a whole spectrum of food approaches ranging from avoidance (high HA) to urgency (high NS) may rely on different temperament profiles.

\section{Conclusions}

An intriguing association between temperament, namely, high HA, and food approach (under eating) emerged. Longitudinal studies may want to evaluate whether these traits represent a risk factor for the development of EDs.

\section{Conflict of Interests}

The authors declare that there is no conflict of interests regarding the publication of this paper.

\section{References}

[1] C. R. Cloninger, D. M. Svrakic, and T. R. Przybeck, "A psychobiological model of temperament and character," Archives of General Psychiatry, vol. 50, no. 12, pp. 975-990, 1993.

[2] K. L. Klump, M. Strober, C. M. Bulik et al., "Personality characteristics of women before and after recovery from an eating disorder," Psychological Medicine, vol. 34, no. 8, pp. 1407-1418, 2004.

[3] L. R. R. Lilenfeld, "Personality and temperament," Current Topics in Behavioral Neurosciences, vol. 6, pp. 3-16, 2011.

[4] S. Fassino, G. Abbate-Daga, F. Amianto, P. Leombruni, S. Boggio, and G. G. Rovera, "Temperament and character profile of eating disorders: A controlled study with the temperament and character inventory," International Journal of Eating Disorders, vol. 32, no. 4, pp. 412-425, 2002.

[5] P. K. Keel and K. J. Forney, "Psychosocial risk factors for eating disorders," International Journal of Eating Disorders, vol. 46, no. 5, pp. 433-439, 2013.

[6] G. Abbate-Daga, C. Gramaglia, G. Malfi, A. Pierò, and S. Fassino, "Eating problems and personality traits. An Italian pilot study among 992 high school students," European Eating Disorders Review, vol. 15, no. 6, pp. 471-478, 2007.

[7] W. H. Kaye, J. L. Fudge, and M. Paulus, "New insights into symptoms and neurocircuit function of anorexia nervosa," Nature Reviews Neuroscience, vol. 10, no. 8, pp. 573-584, 2009.

[8] P. Pisani, F. Faggiano, V. Krogh, D. Palli, P. Vineis, and F. Berrino, "Relative validity and reproducibility of a food frequency questionnaire for use in the Italian EPIC centres," International Journal of Epidemiology, vol. 26, supplement 1, pp. S152-S160, 1997.

[9] Società Italiana di Nutrizione Umana, "Livelli di assunzione raccomandati di energia e nutrienti per la popolazione Italiana," in Proceedings of the Acts of 25th National Meeting, 2012.

[10] E. Stice, J. Ng, and H. Shaw, "Risk factors and prodromal eating pathology," Journal of Child Psychology and Psychiatry and Allied Disciplines, vol. 51, no. 4, pp. 518-525, 2010. 


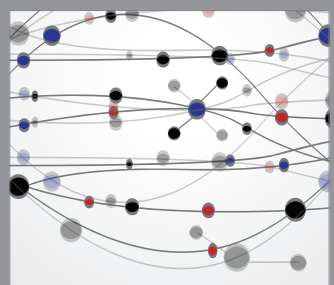

The Scientific World Journal
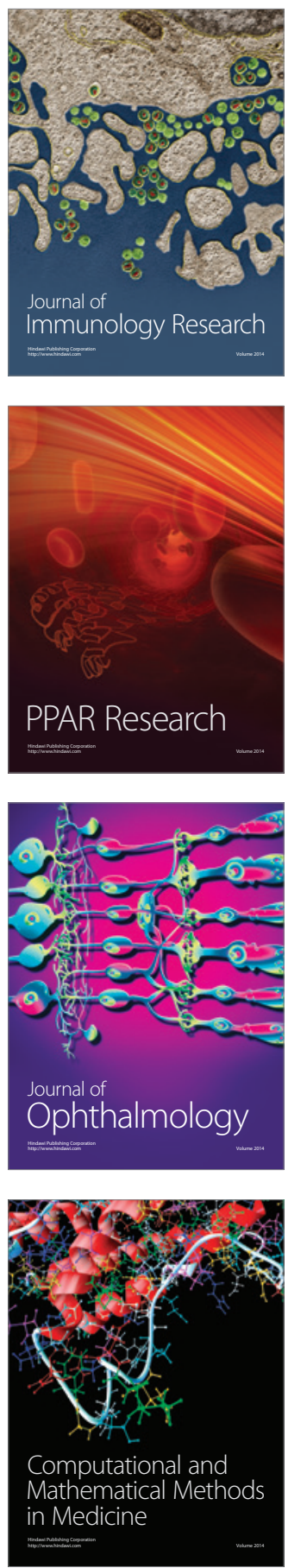

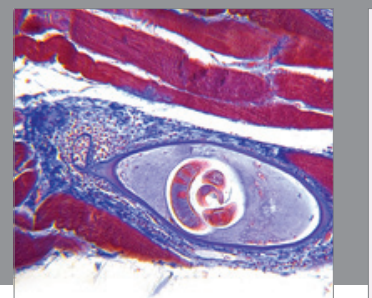

Gastroenterology

Research and Practice
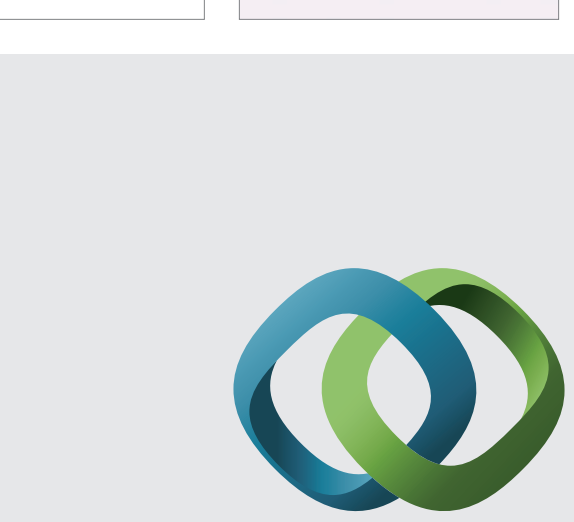

\section{Hindawi}

Submit your manuscripts at

http://www.hindawi.com
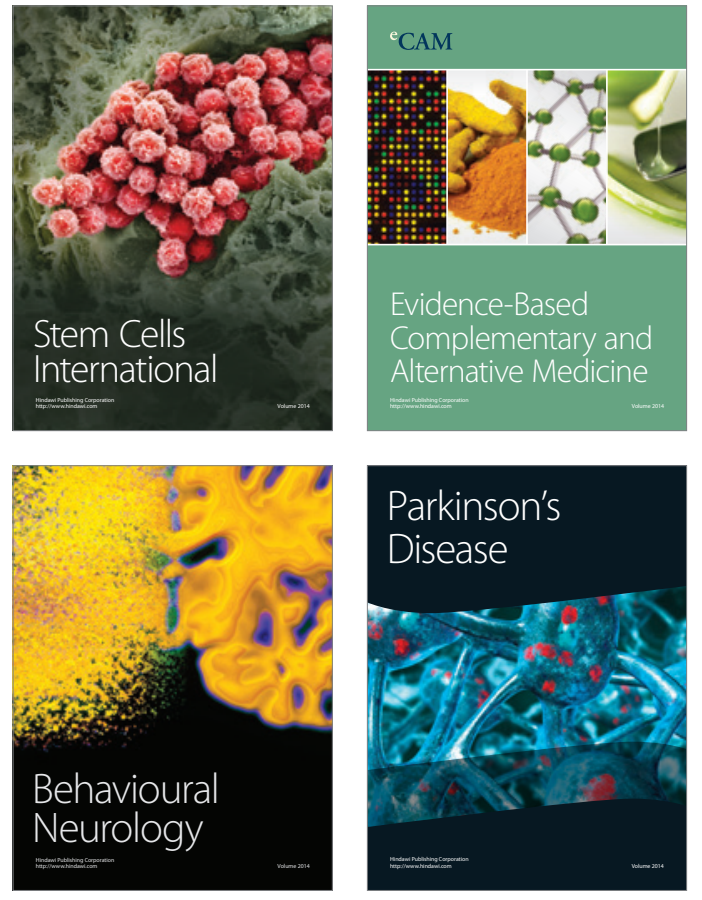
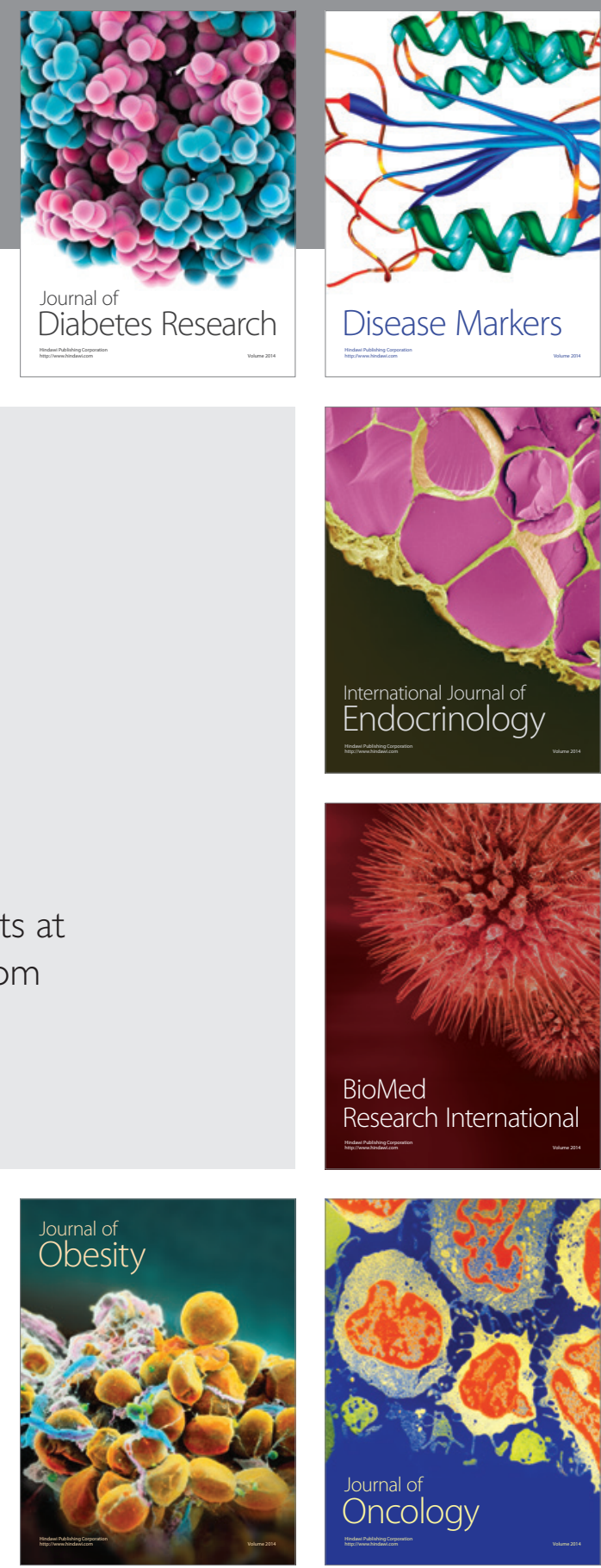

Disease Markers
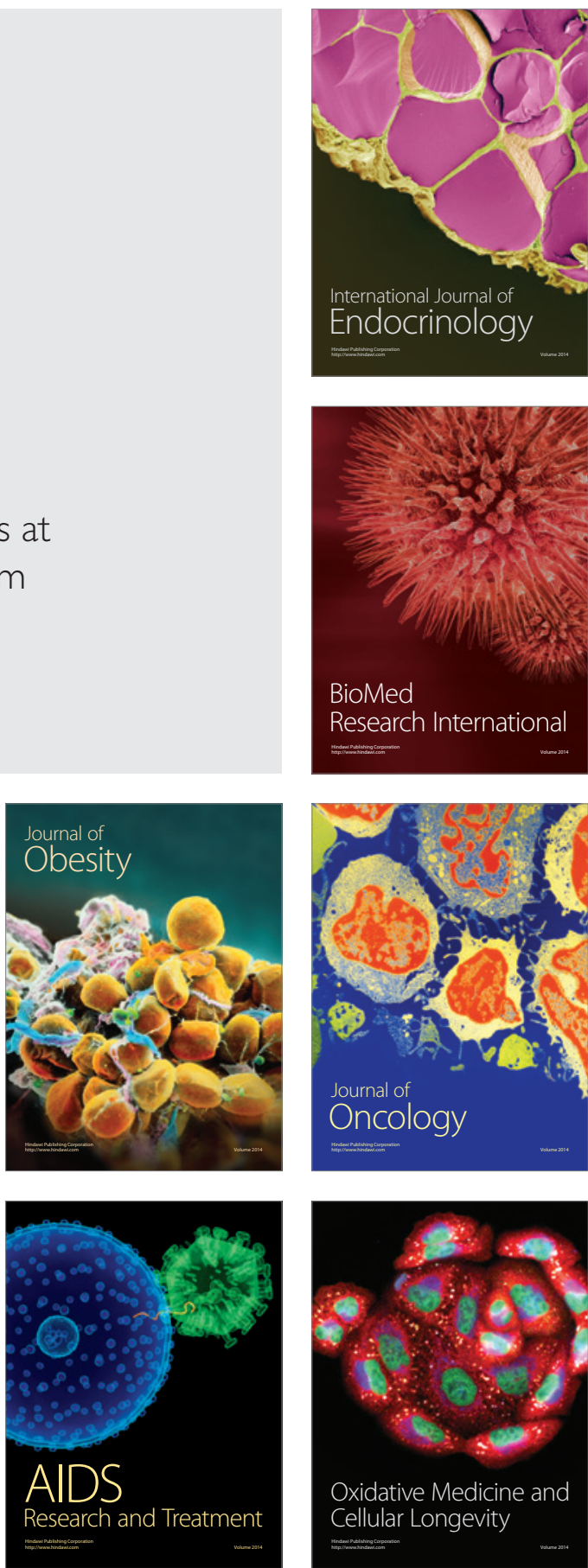